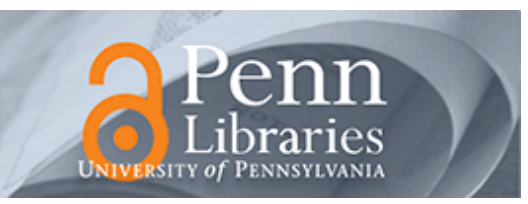

University of Pennsylvania

ScholarlyCommons

May 1998

\title{
Experimental Implementation of a "Target Dynamics" Controller on a Two-link Brachiating Robot
}

\author{
Jun Nakanishi \\ Nagoya University \\ Toshio Fukuda \\ Nagoya University \\ Daniel E. Koditschek \\ University of Pennsylvania, kod@seas.upenn.edu
}

Follow this and additional works at: https://repository.upenn.edu/ese_papers

\section{Recommended Citation}

Jun Nakanishi, Toshio Fukuda, and Daniel E. Koditschek, "Experimental Implementation of a "Target Dynamics" Controller on a Two-link Brachiating Robot", . May 1998.

Copyright 1998 IEEE. Reprinted from Proceedings of the IEEE International Conference on Robotics and Automation, Volume 1, 1998, pages 787-792.

This material is posted here with permission of the IEEE. Such permission of the IEEE does not in any way imply IEEE endorsement of any of the University of Pennsylvania's products or services. Internal or personal use of this material is permitted. However, permission to reprint/republish this material for advertising or promotional purposes or for creating new collective works for resale or redistribution must be obtained from the IEEE by writing to pubs-permissions@ieee.org. By choosing to view this document, you agree to all provisions of the copyright laws protecting it.

NOTE: At the time of publication, author Daniel Koditschek was affiliated with the University of Michigan. Currently, he is a faculty member in the Department of Electrical and Systems Engineering at the University of Pennsylvania.

This paper is posted at ScholarlyCommons. https://repository.upenn.edu/ese_papers/371

For more information, please contact repository@pobox.upenn.edu. 


\title{
Experimental Implementation of a "Target Dynamics" Controller on a Two-link Brachiating Robot
}

\author{
Abstract \\ We report on our recent empirical success in the study of a two-link brachiating robot. The "target \\ dynamics" controller developed in our previous work (1997) is implemented on a physical system in our \\ laboratory. The swing locomotion and swing-up behavior of the robot as well as continuous locomotion \\ have been successfully attained. The experimental results illustrate the effectiveness of our control \\ strategy.

\section{Comments} \\ Copyright 1998 IEEE. Reprinted from Proceedings of the IEEE International Conference on Robotics and \\ Automation, Volume 1, 1998, pages 787-792. \\ This material is posted here with permission of the IEEE. Such permission of the IEEE does not in any way \\ imply IEEE endorsement of any of the University of Pennsylvania's products or services. Internal or \\ personal use of this material is permitted. However, permission to reprint/republish this material for \\ advertising or promotional purposes or for creating new collective works for resale or redistribution must \\ be obtained from the IEEE by writing to pubs-permissions@ieee.org. By choosing to view this document, \\ you agree to all provisions of the copyright laws protecting it. \\ NOTE: At the time of publication, author Daniel Koditschek was affiliated with the University of Michigan. \\ Currently, he is a faculty member in the Department of Electrical and Systems Engineering at the \\ University of Pennsylvania.
}


Proceedings of the 1998 IEEE

International Conference on Robotics \& Automation

Leuven, Belgium • May 1998

\title{
Experimental Implementation of a "Target Dynamics" Controller on a Two-link Brachiating Robot
}

\author{
Jun Nakanishi*, Toshio Fukuda ${ }^{\dagger}$ and Daniel E. Koditschek ${ }^{\ddagger}$ \\ * Dept. of Micro System Engineering, Nagoya University, Nagoya, Aichi 464-01, Japan \\ ${ }^{\dagger}$ Center for Cooperative Research in Advanced Science and Technology, Nagoya University, Nagoya, Aichi 464-01, Japan \\ ${ }^{\ddagger}$ Dept. of Electrical Engineering and Computer Science, The University of Michigan, Ann Arbor, MI 48109-2110, USA
}

\begin{abstract}
We report on our recent empirical success in the study of two-link brachiating robot. The "target dynamics" controller developed in our previous work is implemented on a physical system in our laboratory. The swing locomotion and swing up behavior of the robot as well as continuous locomotion have been successfully attained. The experimental results illustrate the relevance of our control strategy.
\end{abstract}

\section{Introduction}

This paper presents our recent experimental work on the control of a two-link brachiating robot (see Figure 1). Robot brachiation was initially achieved by the second author and Saito $[13,12]$ using learning methods ${ }^{1}$. In our previous work [8], we proposed a new control algorithm, developed via "target dynamics" methods. Inspired by the pendulum-like motion of an ape's brachiation, the task is encoded as an output of an appropriately chosen target dynamical system-a harmonic oscillator. Preliminary numerical studies and analysis illustrated that the proposed controller solved the "ladder", "swing up" and "rope" problems as defined in [8].

We are interested in dynamical dexterity [4] that requires dynamical interaction with an unactuated environment to achieve a designated task, such as juggling, hopping and other tasks concerned with the requirement of regulation of kinetic as well as potential energy. Brachiation takes an interesting place in this realm of dynamically dexterous robotics including dexterous manipulation $[1,2,3,6,11]$, legged locomotion $[5,10,14,15]$ and underactuated mechanisms [17]. We hope to gain insight into control of dynamically dexterous behavior through our study of robot brachiation.

In this paper, we present the successful experimental implementation of the proposed controller on a physical system. In particular, we use the two-link brachiating robot built by Saito [13], where the original controller hardware is replaced, as discussed in Section 3.1.

Currently, our experimental success encompasses a number of brachiation tasks starting from a variety of different initial "hand" positions. We have achieved swing locomotion in the ladder problem, where both hands are initially on the ladder; various swing-up behaviors from a suspended posture, where only one hand

\footnotetext{
${ }^{1}$ Recall that our approach using target dynamics from nonlinear control point of view is rather different from their learning approach as discussed in [7].
}

is initially on the ladder; and continuous locomotion over several rungs, where the robot starts with either one or both hands on the ladder.

However, due to the structure of the gripper, the rope problem cannot be experimentally carried out with this robot. The empirical success presented herein demonstrates that our algorithm is practically relevant in spite of its many simplifications and disregard of various physical effects.

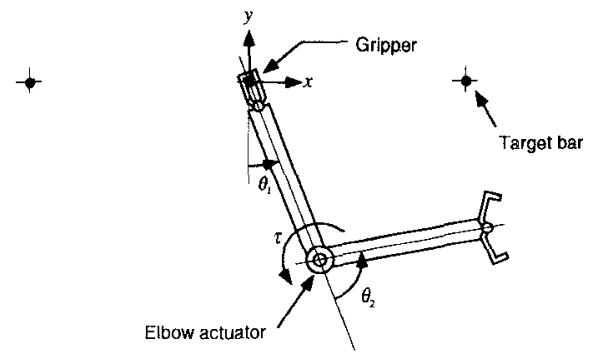

Figure 1: A two-link brachiating robot

\section{Review of Target Dynamics Method}

In this section, we briefly review our control strategy for a simplified point mass lossless model of a two-link brachiating robot. A detailed development of the controller can be found in [8]. The notion of the "target dynamics" is a particular instance of input/output linearization. Specifically, brachiation is encoded as the output of a target dynamical system a harmonic oscillator, which we will force the robot to mimic.

\subsection{Task Encoding: Target Dynamics}

It is traditional in the underactuated robot control literature to use a linearizing feedback to force an output of a system to track some reference trajectory $r_{d}(t)$. We find it more useful to mimic a reference dynamical system. Consider the dynamics of the two-link brachiating robot which take the form of a standard two-link planar manipulator

$$
\dot{T} q=\mathcal{L}(T q, \tau)=\left[\begin{array}{c}
\dot{q} \\
\left.M^{-1}\left(-B-k+\left[\begin{array}{l}
0 \\
\tau
\end{array}\right]\right)\right]
\end{array}\right.
$$


where, $T q=[q, \dot{q}]^{T}, M$ is the inertia matrix, $B$ is the Coriolis/centrifugal vector, $k$ is the gravity vector, and $\tau$ is the joint torque.

According to the biomechanics literature [9], slow brachiation of apes resembles the motion of a pendulum. Although the ape's moment of inertia varies during the swing according to its change of posture, the motion of a simplified pendulum gives a fairly good approximation. Motivated by this pendulum-like motion of brachiation, we choose to encode the task in terms of the even simpler linearized version,

$$
y=T x=\left[\begin{array}{l}
x \\
\dot{x}
\end{array}\right], f_{\omega}(T x)=\left[\begin{array}{cc}
0 & 1 \\
-\omega^{2} & 0
\end{array}\right] T x,
$$

which will serve as the target dynamical system.

Now, we will find it useful to introduce a submersion arising from the change of coordinates from joint space. to polar coordinates on $\mathbb{R}^{2}$,

$$
\left[\begin{array}{l}
r \\
\theta
\end{array}\right]=\tilde{q}=\bar{g}(q)=\left[\begin{array}{c}
l \sqrt{2\left(1+\cos \theta_{2}\right)} \\
\theta_{1}+\frac{1}{2} \theta_{2}
\end{array}\right] .
$$

Specifically, we will take the second component of (3)

$$
x=h(q):=\theta=\theta_{1}+\frac{1}{2} \theta_{2} .
$$

Using the input/output linearizing scheme, the torque input realizing the characteristics of the target dynamical system (2) is

$$
\begin{aligned}
\tau:= & \left(D_{q} h\left[\begin{array}{l}
n_{12} \\
n_{22}
\end{array}\right]\right)^{-1}\left[-\omega^{2} \theta-\left(\dot{D_{q}} h\right) \dot{q}+D_{q} h_{2} M^{-1}(B+k)\right] \\
= & \frac{1}{n_{12}+\frac{1}{2} n_{22}}\left[-\omega^{2}\left(\theta_{1}+\frac{1}{2} \theta_{2}\right)+\left(n_{11}+\frac{1}{2} n_{21}\right)\left(B_{1}+k_{1}\right)\right] \\
& +B_{2}+k_{2}
\end{aligned}
$$

where, $n_{i j}$ denotes each component of $M^{-1}$. Note that

$$
D_{q} h\left[\begin{array}{c}
n_{12} \\
n_{22}
\end{array}\right]=n_{12}+\frac{1}{2} n_{22}=\frac{m_{1} l^{2}}{2 \operatorname{det}(M)} \neq 0
$$

i.e., the invertibility condition of the first term in (5) is satisfied in the particular setting of concern.

\section{Experimental Setup and Mod- elling}

\subsection{Experimental Setup}

This section describes our experimental system. We use the two-link brachiating robot originally developed by Saito [13]. We have, however, replaced the controller hardware such as the computer, input-output devices and motor driver circuits. Figure 2 depicts the configuration of the experimental setup. The length of each arm is $0.5 \mathrm{~m}$ and the total weight of the robot is about $4.8 \mathrm{~kg}$.

Originally, in Saito's experimental setup, a personal computer equipped with $I / O$ devices was used to control the robot. We have replaced it with a VME bus board computer, MVME 167 (Motorola, CPU MC68040, 33MHz), with a real-time operating system,

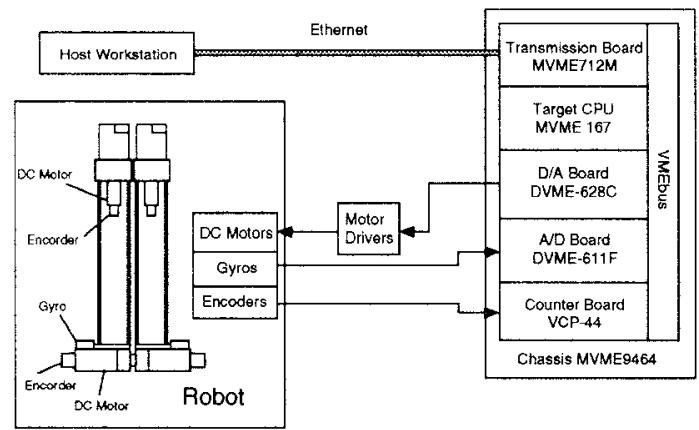

Figure 2: The experimental setup of the two-link brachiating robot.

VxWorks 5.1 and VME bus based $\mathrm{I} / \mathrm{O}$ devices. The control law is evaluated exactly at a rate of $500 \mathrm{~Hz}$.

The elbow joint is actuated by two DC motors with harmonic gears (Harmonic Drive Systems, RH-14-6002). The stator of each motor is fixed to a link, and their rotor shafts are directly connected to each other. As a consequence, we can achieve a total rotational speed at the elbow which is two times faster than the case where there is only one motor. This was necessary since the rated rotational speed of these motors is $360 \mathrm{deg} / \mathrm{sec}$, while we require that the rotational speed of the elbow be over $600 \mathrm{deg} / \mathrm{sec}$. An additional benefit of the symmetrical structure of this design is better overall balance in the mechanism. Each gripper is equipped with a DC motor which opens and closes it.

The angle of the first joint is measured by integrating its angular velocity, which is in turn obtained through a gyro (Murata, ENV-05S) attached to the arm. The angle of the second joint and the opening angle of the gripper are measured using optical encoders.

\subsection{Modelling of the Robot}

A precise model of the robot is necessary for our control algorithm since it requires exact knowledge of the dynamics of the plant. In our previous work, a simplified point mass, lossless model is used assuming that the point mass is located at the end of each link and that the torque of the second joint can be directly controlled. In this section, we introduce a more practical model for the implementation of the proposed controller on the physical system.

\subsubsection{Mathematical Model of the Robot}

We introduce a model, depicted in Figure 3, of the two-link brachiating robot used in our experiments. The dynamical equations used to model the robot are

$$
\dot{T} q=\mathcal{L}\left(T q, v_{r}\right)
$$

where

$$
\begin{aligned}
& q=\left[\theta_{1}, \theta_{2}\right]^{T} \in \mathcal{Q}, T q=[q, \dot{q}]^{T} \in T \mathcal{Q}
\end{aligned}
$$

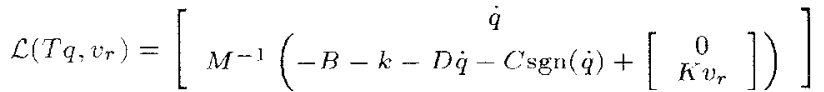

$$
\begin{aligned}
& m_{11}=m_{1} l_{c 1}{ }^{2}+I_{1}+m_{2}\left(l_{1}^{2}+2 l_{1} l_{c 2} \cos \theta_{2}+l_{c 2}{ }^{2}\right)+I_{2} \\
& m_{12}=m_{21}=m_{2}\left(l_{c 2}^{2}+l_{1} l_{c 2} \cos \theta_{2}\right)+I_{2} \\
& m_{22}=m_{2} l_{c 2}{ }^{2}+I_{2}
\end{aligned}
$$




$$
\begin{gathered}
B(q, \dot{q})=-m_{2} l_{1} l_{c 2} \sin \theta_{2}\left[\begin{array}{c}
2 \dot{\theta_{1}} \dot{\theta_{2}}+{\dot{\theta_{2}}}^{2} \\
-\dot{\theta_{1}}{ }^{2}
\end{array}\right] \\
k(q)=\left[\begin{array}{c}
m_{1} g l_{c 1} \sin \theta_{1}+m_{2} g\left(l_{1} \sin \theta_{1}+l_{c 2} \sin \left(\theta_{1}+\theta_{2}\right)\right) \\
m_{2} g l_{c 2} \sin \left(\theta_{1}+\theta_{2}\right)
\end{array}\right] \\
D=\operatorname{diag}\left\{d_{i}\right\}, \quad C=\operatorname{diag}\left\{c_{i}\right\},
\end{gathered}
$$

where, $m_{i}$ and $I_{i}$ are the mass and the moment of inertia of each link respectively, and $l_{i}$ is the link length. The center of mass of each link is located on the center line which passes through adjacent joints at a distance $l_{c i} . C_{i}$ and $D_{i}$ denote the coulomb and viscous friction coefficients respectively. We assume that the elbow actuator produces torque proportional to a voltage command, $v_{r}$, sent to a driver as $\tau=K v_{r}$, where $K$ is a positive constant.

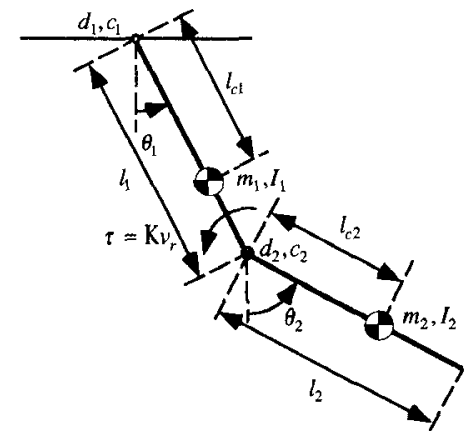

Figure 3: The mathematical model of the two-link brachiating robot used for the experimental implementation of the controller.

It is generally known that DC motors with harmonic gear mechanisms bear complicated nonlinear characteristics, which are considered difficult to model. However, for simplicity, we model the dynamics using only viscous and coulomb friction and rotor inertia. As the results of parameter identification presented in the following section suggest, the model we offer here fits the dynamics of the physical system fairly well.

\subsubsection{Parameter Identification}

We need to identify the dynamical parameters corresponding to the robot's Lagrangian dynamics. We initially considered an off-line least squares estimation method with torque filtering [16], but were unable to obtain a good estimate of the parameter set with this scheme. In consequence, we resorted to a rather simple identification procedure, where the inertia parameters are obtained either via direct measurement or from the manufacturer's data, and the preliminary estimate of the friction coefficients are obtained from the natural dissipation of the system. These parameters were refined iteratively by comparing step and sinusoid responses obtained experimentally to those generated by simulations using the "best" parameters. In this comparison, we considered step response with various amplitude as well as sinusoid response with various amplitude and frequencies. The results of the parameter identification are listed in Table 1. Here, the mass of the two motors at the elbow joint is included in the first link to make clear the correspondence to the simplified model used in our previous study [8], where the mass ratio $\frac{m_{1}}{m_{2}}=3$. However, we could also derive an equivalent model having symmetry in the link parameters since there is redundancy in the inertia parameters. The efficacy of this parameter identification approach is illustrated in Figure 4 which shows examples of the comparison between experimental runs and simulations using the parameters of Table 1.

\begin{tabular}{|c|c|c|c|}
\hline \multicolumn{2}{|c|}{ Description } & $i=1$ & $i=2$ \\
\hline \hline Mass & $m_{i}(\mathrm{~kg})$ & 3.499 & 1.232 \\
\hline Moment of inertia & $I_{i}\left(\mathrm{kgm}^{2}\right)$ & 0.090 & 0.033 \\
\hline Link length & $l_{i}(\mathrm{~m})$ & 0.50 & 0.50 \\
\hline Location of CG & $l_{c i}(\mathrm{~m})$ & 0.414 & 0.333 \\
\hline Viscous friction & $D_{i}(\mathrm{Nm} / \mathrm{s})$ & 0.02 & 0.14 \\
\hline Coulomb friction & $C_{i}(\mathrm{Nm})$ & 0.02 & 0.45 \\
\hline Torque constant & $K(\mathrm{Nm} / \mathrm{V})$ & \multicolumn{2}{|c|}{1.752} \\
\hline
\end{tabular}

Table 1: The dynamical parameters of the robot obtained by the procedure described in Section 3.2.2.
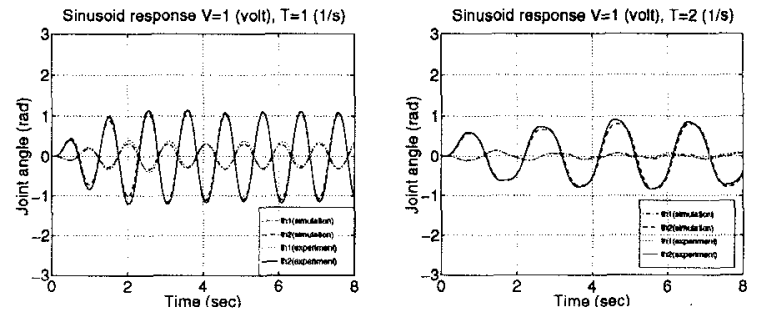

Figure 4: Examples of the comparison between experimental runs and simulations. Left: voltage command $v_{r}=\sin (2 \pi t)$, right: voltage command $v_{r}=\sin (\pi t)$. These plots show close matching between the numerical simulations using the obtained model and experiments.

\subsubsection{Modification of the Controller}

The controller (5) designed for the simplified model (1) is slightly modified for the model of the robot (7) introduced above. The modified control law, realizing the target dynamics, can be given in terms of the voltage command to the motor driver as follows,

$$
\begin{aligned}
v_{r}:= & \frac{1}{K}\left(D_{q} h\left[\begin{array}{l}
n_{12} \\
n_{22}
\end{array}\right]\right)^{-1}\left[-\omega^{2} \theta-\left(\dot{D_{q}} h\right) \dot{q}\right. \\
& +D_{q} h M^{-1}(B+k+D \dot{q}+C \operatorname{sgn}(\dot{q})] \\
= & \frac{1}{K\left(n_{12}+\frac{1}{2} n_{22}\right)}\left[-\omega^{2}\left(\theta_{1}+\frac{1}{2} \theta_{2}\right)\right. \\
& \left.+\left(n_{11}+\frac{1}{2} n_{21}\right)\left(B_{1}+k_{1}+d_{1} \dot{\theta_{1}}+c_{1} \operatorname{sgn}\left(\dot{\theta_{1}}\right)\right)\right] \\
& +\frac{1}{K}\left[B_{2}+k_{2}+d_{2} \dot{\theta_{2}}+c_{2} \operatorname{sgn}\left(\dot{\theta_{2}}\right)\right]
\end{aligned}
$$

where, $n_{i j}$ denotes each component of $M^{-1}$.

Note that the closed loop dynamics of the system does not strictly admit a reverse time symmetry [8], since the uncancelled friction terms of the first joint enter the dynamics of the unactuated degree of freedom. Under these circumstances, numerical simulation suggests that the desired brachiation can be achieved by allowing a tolerance for the gripper position at the capture of the 
bar. In practice, we have experimentally found that model mismatch and disturbances caused by cables seem to affect behavior of the robot rather considerably.

\section{Experiment}

We present results of the experimental implementation of the proposed controller in order to validate our control strategy.

\subsection{Ladder Problem}

This section considers the ladder problem brachiation on a set of evenly spaced bars at the same height. In the experimental setting, the next bar is located at a distance of $0.6 \mathrm{~m}$.

\subsubsection{Implementation of the Controller}

As discussed in [8], the symmetry property of neutral orbits solves that ladder problem. We need to choose $\omega$ in the target dynamics (2) for a given ladder distance, $d^{*}$. For our experimental setting, the approximated value of $\omega$ is calculated to be $\omega=3.36$ using the numerical procedure presented in [7].

This first attempt to implement the controller unfortunately resulted in failure. Swing motion close to the desired behavior was achieved, but the gripper did not come close enough to the target bar to catch it. Therefore, we have found in necessary to introduce some refinements in order to achieve successful brachiation.

In practice, we need to consider the time lag in opening the gripper when the robot initiates locomotion, something not taken into account in the analytical work. It takes approximately 0.08 to 0.1 seconds to release the bar after the command to open the gripper is sent. Empirically, we have observed that this time affects the swing behavior of the robot. As a result, we choose to send the open command of the gripper 0.08 seconds before the target dynamics controller is turned on.

An additional component contributing to the failure we have experienced is presumably the model mismatch. Therefore, we tune the parameters of the model manually so that the robot successfully achieves the desired brachiation. Some experience is helpful in the refinement of the parameter. We choose to use $m_{1}=$ $3.39, m_{2}=1.30, c_{2}=0.73$ and $d_{2}=0.33$ instead of the values in Table 1 for the ladder problem.

\subsubsection{Experimental Results}

Now we present experimental results of the ladder problem. The actual movement of the robot is depicted in Figure 5, while the joint trajectories and the voltage commands sent to the driver are shown in Figure 6. The mean locomotion time of ten runs is 0.973 seconds with \pm 0.015 second error, which is very close to its analytically calculated value, $t=\frac{\pi}{\omega}=0.935$ seconds.

Notice that the symmetry of the neutral orbit is not perfectly attained in the motion of the robot. We have found in numerical simulation that this may be a result of refining the model parameters in order to achieve successful locomotion.

\subsection{Swing up Problem}

The swing up problem considers the task of swinging up from the suspended posture at rest and catching the next bar. To accomplish this task it is necessary not

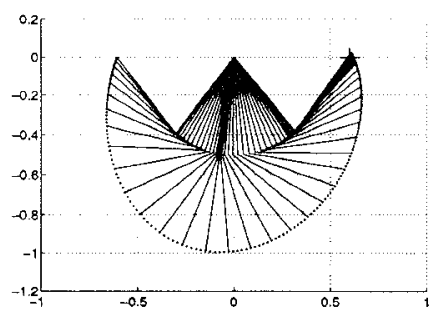

Figure 5: Movement of the robot. The target bar is located at a distance of $0.6 \mathrm{~m}$ marked by the "+".
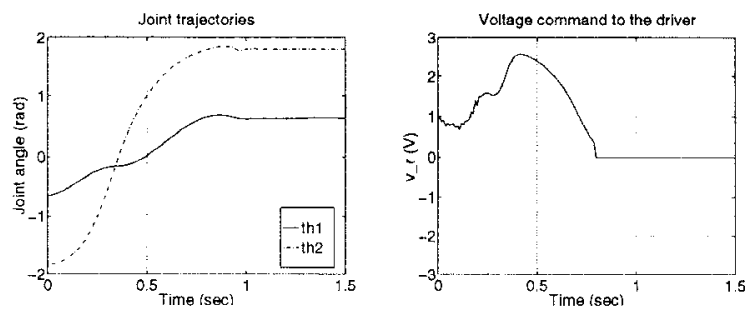

Figure 6: The experimental results of the ladder problem. Left: Joint trajectories, Right: Voltage command to the motor driver

only to pump up the energy, but also to control the arm position at the capture of the next bar. We begin by briefly reviewing our strategy for the swing up problem as discussed in [8] and then present experimental results.

\subsubsection{Review of the Swing up Controller}

As we have mentioned, swing up requires energy pumping in a suitable fashion. In order to introduce a limit cycle, the target dynamics are modified as follows,

$$
\begin{gathered}
\dot{T} x=\left[\begin{array}{cc}
0 & 1 \\
-\omega^{2} & -K_{e}\left(\bar{E}-\bar{E}^{*}\right)
\end{array}\right] T x:=f_{\bar{E}^{*}}(T x) \\
\text { where, } \quad x=\theta=\theta_{1}+\frac{1}{2} \theta_{2} \text { as defined in }(4) \\
K_{e}: \text { a positive constant } \\
\bar{E}:=\frac{1}{2} \dot{\theta}^{2}+\frac{1}{2} \omega^{2} \theta^{2}: \text { "pseudo energy" } \\
\bar{E}^{*}: \text { the desired pseudo energy level }
\end{gathered}
$$

To achieve this target dynamics, the control law is formulated for the experimental system as

$$
\begin{aligned}
v_{r}:= & \frac{1}{K}\left(D_{q} h\left[\begin{array}{c}
n_{12} \\
n_{22}
\end{array}\right]\right)^{-1}\left[-\omega^{2} \theta-K_{e}\left(\bar{E}-\bar{E}^{*}\right) \dot{\theta}\right. \\
& \left.-\left(D_{q} h\right) \dot{q}+D_{q} h M^{-1}(B+k+D \dot{q}+C \operatorname{sgn}(\dot{q}))\right] \\
= & \frac{1}{K\left(n_{12}+\frac{1}{2} n_{22}\right)}\left[-\omega^{2}\left(\theta_{1}+\frac{1}{2} \theta_{2}\right)\right. \\
& -K_{e}^{r}\left(\bar{E}-\bar{E}^{*}\right)\left(\dot{\theta_{1}}+\frac{1}{2} \dot{\theta_{2}}\right) \\
& \left.+\left(n_{11}+\frac{1}{2} n_{21}\right)\left(B_{1}+k_{1}+d_{1} \dot{\theta_{1}}+c_{1} \operatorname{sgn}\left(\dot{\theta_{1}}\right)\right)\right] \\
& +\frac{1}{K}\left[B_{2}+k_{2}+d_{2} \dot{\theta_{2}}+c_{2} \operatorname{sgn}\left(\dot{\theta_{2}}\right)\right]
\end{aligned}
$$

The time derivative of the pseudo energy, $\bar{E}$, along the motion implies the convergence of $\bar{E} \rightarrow E^{*}$, suggesting that this control law achieves a stable limit cycle with 
respect to $\theta$ coordinates whose trajectory is characterized by $\frac{1}{2} \dot{\theta}^{2}+\frac{1}{2} \omega^{2} \theta^{2}=\bar{E}^{*}$.

\subsubsection{Experimental Results}

In order to achieve the task, we need to bring the effective actuated portion of the state, $\theta$, to the right pseudo energy level, while simultaneously ensuring the unactuated degree of freedom, $r$, coincide with the regulated length between the bars, $d^{*}$. As we have discussed in [8], the procedure in choosing $K_{e}$ is somewhat ad hoc. Some experience is helpful in determining the proper choice of the parameters. Since choosing $K_{e}$ large yields "chaotic" motion, we prefer to choose $K_{e}$ small. However, this results in relatively slow swing up motion. Numerical studies suggest that some particular choices of larger $K_{e}$ may result in robot trajectories which go through the next bar's position after a few of swings. Such motion allow for faster swing up times, as long as the robot catches the bar when the gripper's position coincides with that of the target bar. "Chaotic" motion in the swing behavior is observed if we let the robot keep swinging without grasping the bar at that time.

What follows is a presentation of the different swing up behaviors resulting from changes in the rate of energy pumping, as characterized by $K_{e}$. The distance of the bars is $0.6 \mathrm{~m}$. We consider three cases where $K_{e}=0.03,0.47$ and 0.9 . These parameters are chosen manually based on our experience. In order to successfully swing up, we have found it necessary to slightly modify the desired pseudo energy level and some of the model parameters. We choose $\bar{E}^{*}=1.1 \bar{E}_{n o m}^{*}$, where $\bar{E}_{n o m}^{*}=\frac{1}{2} \omega^{2}\left(\frac{\pi}{2}\right)^{2}$, and $m_{1}=3.39, m_{2}=1.30$ instead of the values in Table 1 . The initial direction of the swing motion depends solely upon the initial states of the system since the motion of the robot is governed by the closed loop dynamics. Only small deviation from the origin on the phase plane determines this direction. Thus, we introduce an impulse-like initial torque before the controller is turned on so that the robot starts its swing motion in the desired direction at every run.

Slow Swing up $\left(K_{e}=0.03\right)$ Consider the case where $K_{e}=0.03$. Figure 7 shows the joint trajectory and the voltage command to the motor driver. The mean time of ten runs for this slow swing up behavior is 7.474 seconds with \pm 0.080 second error.
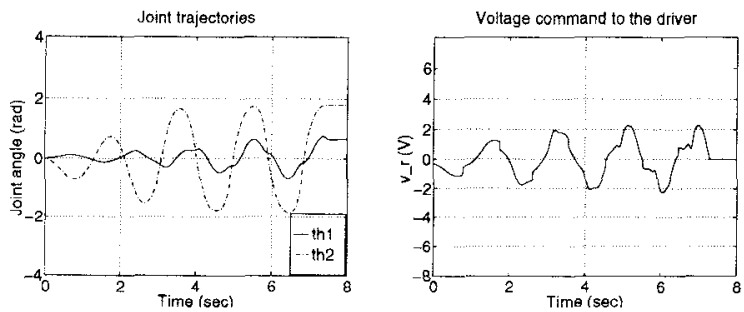

Figure 7: Experimental results of slow swing up behavior $\left(K_{e}=0.03\right)$. Left: Joint trajectories, right: Voltage command to the motor driver. The robot captures the bar when $t \sim 7.5$ seconds.

Fast Swing up $\left(K_{e}=0.47\right)$ Consider the case where $K_{e}=0.47$. Figure 8 shows the joint trajectory and the voltage command to the motor driver. This choice $K_{e}$ yields relatively fast swing up. The mean swing up time of ten runs for this swing up is 3.843 seconds with \pm 0.146 second error.
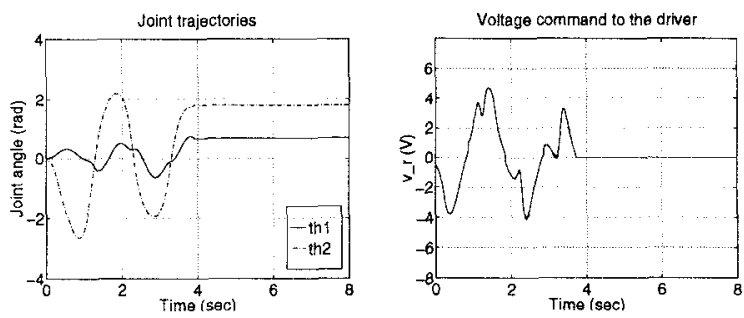

Figure 8: Experimental results of fast swing up behavior $\left(K_{e}=0.47\right)$. Left: Joint trajectories, right: Voltage command to the motor driver. The robot captures the bar when $t \sim 3.8$ seconds.

Rather Faster Swing up $\left(K_{e}=0.9\right)$ Consider the case where $K_{e}=0.9$. Figure 9 shows the joint trajectory and the voltage command to the motor driver. This choice of $K_{e}$ yields a "rather faster" swing up maneuver. The mean swing up time of ten runs for this movement is 2.913 seconds with \pm 0.025 second error. In this case, the initial impulse-like torque is applied in the opposite direction to the previous two cases in order to start swinging in the CCW direction.
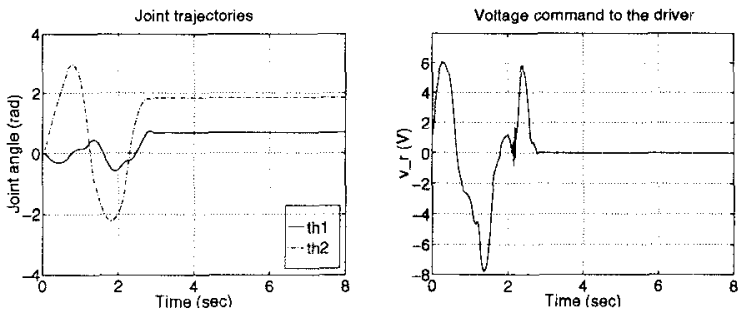

Figure 9: Experimental results of rather faster swing up behavior $\left(K_{e}=0.9\right)$. Left: Joint trajectories, right: Voltage command to the motor driver. The robot captures the bar when $t \sim 2.9$ seconds.

\subsection{Continuous Locomotion}

Here we exhibit the demonstration of continuous locomotion over several rungs of the ladder. Figure 10 depicts continuous locomotion of the robot initiated at the ceiling and moving from left to right. This motion can be considered as the iteration of the ladder trajectory. After each swing, the initial condition is reset, and the function of each arm is switched. Due to the symmetrical structure of the robot, the same model is used in each swing where the configuration of the robot is "flipped over." In Figure 11, we show a picture of continuous locomotion initiated from the suspended posture. This is a combination of the "rather faster" swing up maneuver and the iterated ladder trajectory. First, the robot swings three times--going forth (1) and back (2) to gain momentum, and again swinging forward (3) to catch the bar - with the swing up controller $\left(K_{e}=0.9\right)$ described above. Then the control law is switched into the locomotion controller. 


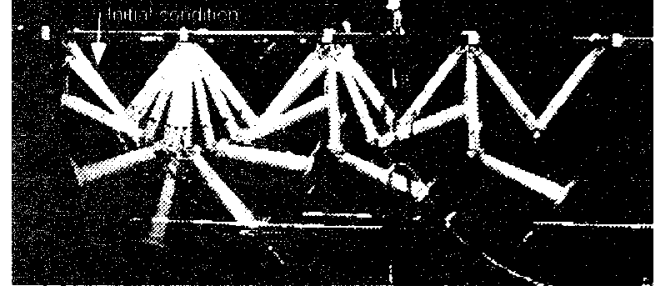

Figure 10: A Picture of continuous locomotion started in the ceiling. The robot iterates brachiation three times moving from left to right.

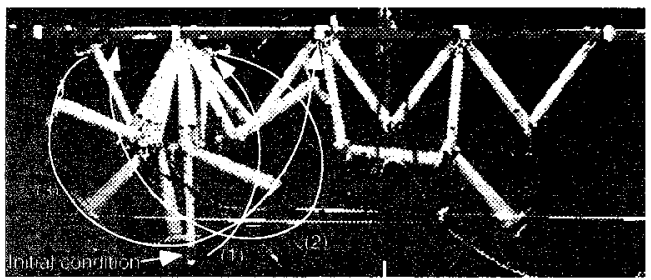

Figure 11: A Picture of continuous locomotion initiated from the suspended posture. The robot moves from left to right. The control law is switched from the swing up controller into the regular locomotion controller after the swing up motion.

In these experiments, we have observed that disturbances caused by the cable, which hangs down from above, can occasionally have a detrimental effect on the robot's motion. In particular, sometimes, the robot has difficulty reaching the bar because of the dragging effect of the cable. Thus, some care has to be taken so that the influence of the cable can be reduced. Nonetheless, we feel that these experimental results demonstrate the relevance of our strategy despite the many practical issues which have not been formally treated, such as model mismatch, inaccuracy of sensors and actuators, and the presence of various disturbances.

\section{Conclusion}

We have presented our empirical success in the implementation of the target dynamics method to the two-link brachiating robot. The proposed algorithm is applied to the ladder and swing up problem. We achieved swing locomotion in the ladder problem and various swing up behaviors with different rates of energy pumping, as characterized by $K_{e}$. We demonstrated continuous locomotion over several rungs of the ladder as well. The experimental success bears out the validity of our control strategy in spite of the presence of model mismatches and physical effects previously unconsidered. Even so, some manual tuning was required to implement our ideas. As such, future work will entail on-line parameter tuning and an adaptive or robust version of the controller.

A formal analysis of our control algorithm still remains to be addressed in order to truly understand how these ideas work. Finally, we are hopeful that, in the long run, our ideas may have wider application to the more general area of dynamically dexterous robotics.

We thank Bill Schwind of the University of Michigan for his help in proofreading.

\section{References}

[1] Andersson, R. L. A Robot Ping-Pong Player: Experiment in Real-Time Intellgent Control. MIT Press, 1988.

[2] Bühler, M., Koditschek, D. E., and Kindlmann, P. J. A family of robot control strategies for intermittent dynamical environments. IEEE Control Systems Magazine, pages 16-22, February 1990.

[3] Burridge, R. R., Rizzi, A. A., and Koditschek, D. E. Toward a systems theory for the composition of dynamically dexterous behaviors. In 7th Internatinal Symposium on Robotics Research, 1995.

[4] Koditschek, D. E. Dynamically dexterous robots. In Spong, M. W., Lewis, F. L., and Abdallah, C. T., editors, Robot Control: Dynamics, Motion Planning and Analysis, pages 487-490. IEEE Press, 1993.

[5] Koditschek, D. E. and Bühler, M. Analysis of a simplified hopping robot. International Journal of Robotics Research, 10(6):587--605, December 1991.

[6] Lynch. K. M. Nonprehensile Robotic Manipulation: Controllability and Planning. PhD thesis, Carnegie Mellon University, The Roboties Institute, March 1996.

[7] Nakanishi, J., Fukuda, T., and Koditschek, D. E. Preliminary analy tical approach to a brachiation robot controller. Technical report: CGR 96-08 / CSE TR 30596, The Univ. of Michigan, EECS Depertment, August 1996.

[8] Nakanishi, J., Fukuda, T., and Koditschek, D. E. Preliminary studies of a second generation

brachiation robot controller. In IEEE International Conference on Robotics and Automation, pages 20502056, April 1997

[9] Preuschoft, H. and Demes, B. Biomechanics of brachiation. In Preuschoft, H., Chivers, D. J., Brockelman, W. Y.. and Creel, N., editors, The Lesser Apes, pages 96-228. Edinburgh University Press, 1984.

[10] Raibert, M. H. Legged Robots that Balance. MIT Press, 1986.

[11] Rizzi, A. A., Whitcomb, L. L., and Koditschek, D. E. Distributed real-time control of a spatial robot juggler. IEEE Computer, pages 12-24, May 1992.

[12] Saito, F. and Fukuda, T. Learning architecture for real robotic systems - extension of connectionist Q-learning for continuous robot control domain. In IEEE International Conference on Robotics and Automation, pages $27-32,1994$.

[13] Saito, F., Fukuda, T., and Arai, F. Swing and locomotion control for a two-link brachiation robot. IEEE Control Systems Magazine, 14(1):5-12, February 1994.

[14] Schwind, W. J. and Koditschek, D. E. Control the forward velocity of the simplified planner hopping robot. In IEEE International Conference on Robotics and $A u$ tomation, pages 691-696, 1995.

[15] Schwind, W. J. and Koditschek, D. E. Characterization of monopod equilibrium gaits. In IEEE International Conference on Robotics and Automation, pages 1986 1992,1997

[16] Slotine, J.-J. E. and Li, W. Applied Nonlinear Control. Prentice-Hall, 1991

[17] Spong, $M$. The swing up control problem for the acrobot. IEEE Control Systems Magazinc, 15(1):49 55, February 1995 . 